East African Medical Journal Vol. 85 No. 5 May 2008

POLIO OUTBREAK RESPONSE IN ETHIOPIA

G. Mesfin, MD, MSc, WHO Medical Surveillance Officer, W.W. Schluter, MD, MSPH, WHO Medical Officer, A. Gebremariam, MD, Paediatric Neurologist, Senior Lecturer, Addis Ababa University, D. Benti, MD, MPH, WHO Medical Surveillance Officer, T. Bedada, BSc, Statistician, WHO Data Manager, WHO Country Office, Addis Ababa, Ethiopia, P.O. Box 3069, UNECA Compound, Addis Ababa, Ethiopia, B. Beyene, BSc, National Polio Laboratory Head, Ethiopian Health Nutrition and Research Institute, Addis Ababa, Ethiopia, A. Yigzaw, MD, MPH, WHO Immunisation Officer, N. Mbakuliyemo, WHO EPI Unit Head, O.A. Babaniyi, MD, WHO Representative for Ethiopia, WHO Country Office, Addis Ababa, Ethiopia, P.O. Box 3069, UN ECA Compound, Addis Ababa, Ethiopia

Request for reprints to: Dr. G. Mesfin, WHO Country Office, Addis Ababa, Ethiopia, P.O. Box 3069, UNECA Compound, Addis Ababa, Ethiopia

\title{
POLIO OUTBREAK RESPONSE IN ETHIOPIA
}

\author{
G. MESFIN, W. SCHLUTER, A. GEBREMARIAM, D. BENTI, T. BEDADA, B. BEYENE, A. YIGZAW, \\ Z. TADDESS, N. MBAKULIYEMO and O. BABANIYI
}

\begin{abstract}
Background: Ethiopia had been polio-free for almost four years until December 2004. However, between December 2004 and February 2006, 24 children were paralysed as a result of infection with wild poliovirus imported from the neighbouring country of Sudan. In response, the country has attempted to document the impact of various response measures on the containment of wild poliovirus transmission.

Objectives: This study aims at systematic and epidemiological assessment of the extent of the outbreak, its determinants, and the lessons learned as well as the implications for future control strategies to interrupt wild poliovirus transmission.

Design: A cross-sectional study design with qualitative and quantitative data collection approaches was used to conduct the epidemiologic assessment.

Subjects: All confirmed wild poliovirus cases, and reported acute flaccid paralysis cases in close proximity to the confirmed polio cases were the study subjects. Child caretakers and health service providers were interviewed as part of the investigation.

Results: Between December 2004 and February 2006, eight children from Tigray Regional State, nine children from Amhara Regional State and seven children from Oromia Regional State were paralysed as a result of infection with wild poliovirus type 1 . Genetic sequencing demonstrated two separate importations to Ethiopia. Risk factors that may have facilitated spread of the outbreak within the country included gaps in vaccination coverage and interruption of the cold chain system, gaps in acute flaccid paralysis surveillance performance, high population mobility, poor environmental sanitation, crowded living conditions and unsafe drinking water. In response to the outbreak, Ethiopia conducted detailed outbreak investigations within two days of confirmation of the index cases. Large-scale, house-to-house vaccination campaigns were also implemented. As a result, the three regions interrupted the wild poliovirus transmission within the regions within one year of confirmation of the index case.

Conclusion: Outbreak response activities were successful in interrupting the imported wild poliovirus transmission in Tigray, Amhara and Oromia Regional States of Ethiopia within a oneyear period of time. In Ethiopia, programme strategies should be intensified to contain further spread and prevent future importation of wild poliovirus. Large-scale immunisation campaigns should reach every child, including those isolated by geography, poverty and security.
\end{abstract}




\section{INTRODUCTION}

The polio eradication initiative is the largest public health initiative in history. In 1988, the World Health Assembly passed a resolution to eradicate polio from the globe by the year 2000 (1-3). The basic strategies for eradicating polio include the following: (i) Immunisation of every child aged less than one year with at least three doses of oral polio vaccine (OPV); (ii) National immunisation days (NIDs) in which all children less than five years of age receive extra doses of OPV on two days separated by at least 28 days; (iii) Surveillance for acute flaccid paralysis (AFP) to identify all reservoirs of wild poliovirus transmission; and (iv) Extensive house-to-house immunisation mopping-up campaigns in the final stages in areas where wild poliovirus transmission persists $(2,3)$. In 1988, worldwide there were an estimated 350,000 polio cases and 125 countries with endemic polio transmission. The effective use of the above noted strategies resulted in the interruption of wild poliovirus (WPV) in most parts of the globe. By late 2002 indigenous WPV transmission had been interrupted in all countries of Africa except Egypt, Niger and Nigeria (4-6). With continued global efforts the number of endemic countries and total number of infected children gradually decreased. By 2003 , there were only 784 polio cases in six endemic countries in the world (Afghanistan, Egypt, India, Niger, Nigeria and Pakistan) (2,3). However, a large outbreak occurred in northern Nigeria with subsequent transmission of WPV to western and then to central African countries. The virus spread east to Chad and the Sudan with clustering of the virus along the eastern part of the Sudan bordering Ethiopia. In 2004, importation of wild poliovirus with re-infection in countries that had previously interrupted transmission increased the number of cases to 1,263 in 18 countries ( 6 endemic and 12 with importations) $(5,6)$.

The implementation of the polio eradication strategies in Ethiopia started in 1996 (4). Ethiopia was polio-free for almost four years from January 2001 until December 2004 (7,8). From December 2004 to February 2006 a total of 24 cases were confirmed from Tigray, Amhara and Oromia Regional States. In 2004, the surveillance performance for acute flaccid paralysis (AFP) at national level was above the targets. As of February 2005, the annualised AEP detection rate was 2.24. However, the percentage of cases with two stool specimens collected within 14 days or stool adequacy was at $76 \%$ (below the $80 \%$ target). Even more of concern was that when the data was disaggregated there were gaps and variations at sub-national levels.

Considering population immunity, the three densely populated regions of the country (Amhara, Oromia and Southern Regions) had the highest number of children susceptible to polio due to suboptimal routine immunisation coverage. Moreover, the last nation-wide polio supplementary immunisation activity (SIA) in Ethiopia was in 2002 (8).

This article, therefore, aims at describing the determinants of polio importation to Ethiopia and evaluates the effectiveness of the response measures as tools for future control measures in the country.

\section{MATERIALS AND METHODS}

To elucidate causes and determinants of the polio outbreak, a rapid epidemiological assessment technique was critical. This was believed to provide the most timely feedback for implementation of control interventions. The study design was crosssectional involving both quantitative and qualitative data collection techniques.

A detailed and systematic epidemiologic investigation was carried out on all detected WPV cases. Additionally persons from the communities and villages in close proximity to the confirmed polio cases were interviewed. Routine immunisation and cold chain status were reviewed at nearby health institutions. Health workers' awareness was evaluated. Active case search was conducted for cases of acute flaccid paralysis both in health facilities and at traditional sites (e.g., holy water sites, traditional healers, etc.). Attitudes and practices of health care providers and child caretakers were reviewed in affected communities.

Selection of study subjects: The current polio outbreak investigation assessed all reported wild poliovirus cases $(n=24)$ from Tigray, Amhara and Oromia regions and a sample of households in close proximity to the confirmed wild polio cases using systematic sampling methods. The first household was chosen randomly by spinning a pen. Subsequent households were chosen by selecting every second household until the desired numbers of households were interviewed. Children aged 12-23 months 
were the primary study group to assess routine EPI coverage in the affected districts and communities of the three regions. For SIA coverage, children one to four years of age were the subjects evaluated from the selected households. When the household members were unwilling to take part in the study or were not at home, the house immediately to the right of the selected household was substituted.

In each surveyed community, a focus group discussion (FGD) was organised to assess the awareness and attitudes of the community members toward conditions of poliomyelitis illness. Each focus group consisted of eight to ten members representing various community groups. Separate semi-structured questionnaires were developed to assess the awareness of health workers and child caretakers. Records were reviewed in all nearby health facilities and at traditional healing sites. The investigators inspected and took notes on the status of the cold chain system in all health facilities of the polio-affected communities.

Field investigations: This were conducted in each of the communities with one or more wild poliovirus cases. The field investigation involved the following assessment levels:

(i) Clinical and neurological evaluations were completed on all confirmed polio cases.

(ii) Medical record review was completed to search for unreported AFP cases and to validate the surveillance activities in the affected areas. AFP surveillance activities were reviewed using quality, timeliness and completeness indicators.

(iii) Child caretakers were interviewed in each surveyed household and focus group discussions were held to assess awareness, parental care seeking behaviors and factors that determine their preferences.

(iv) OPV vaccine coverage was surveyed in order to estimate routine OPV coverage and coverage from the most recent national immunisation days (NIDs). A total of 1,017 children 12-23 months of age were selected using the rapid sampling technique to assess routine OPV coverage. For NIDs coverage, children one to four years of age were targeted in the studied households.
Epi Info software, version 3.2.2 statistical package, (13) was used to enter and analyse data as well as to create dot maps of the confirmed wild poliovirus cases.

\section{RESULTS}

A total of 24 children (10 males, 14 females) were paralysed by laboratory-confirmed wild poliovirus, type-1 infections in Tigray, Amhara and Oromia regions of Ethiopia between December 2004 and February 2006 (Table 1). The index case in Tigray occurred in late December 2004 (Figure 1). Twenty two of the 24 cases (92\%) had paralysis onset during the period February - April and September - November in 2005. During 2006, however, only in Amhara region was there a confirmed polio case, while no cases were detected in the other two regions. Sequencing of the poliovirus isolates demonstrated that while the wild poliovirus isolated in Tigray and Amhara were most closely related to poliovirus type 1 (PIa) isolated in Khartoum Sudan, the poliovirus isolates from the East Harerghe cases, however, were most closely related with virus isolated in 2004 from Red Sea Province of the Sudan (PIb).

Following the index cases (Figure 1) in the two separate importations, cases tended to occur in a time interval that ranged from 7 to 14 days. The outbreak in both importations also lasted for five to six incubation periods. The latest date of onset of paralysis due to the imported wild poliovirus, from the three affected regions was on $1^{\text {st }}$ February 2006.

The median age of children with polio was 54 months (range: 12 to 168 months). Of the 24 wild poliovirus cases, $14(58 \%)$ were aged less than three years, $16(67 \%)$ were aged less than five years and eight (33\%) were aged between 5 to 14 years (Figure 2). Of the 24 patients seven (29\%) had never received oral polio vaccine (OPV), seven (29\%) had received one to two OPV doses and 10 (42\%) had received at least three doses of OPV (Table 2). Moreover four out of the five $(80 \%)$ WPV cases with quadriplegia had received less than three doses of OPV.

Clinical and epidemiological evaluation (Table 2) of the paralysed children confirmed that all 24 cases had paralytic poliomyelitis. Of the 24 confirmed polio cases, $23(95.8 \%)$ had residual flaccid paralysis on follow up examination 60 days after paralysis onset. 
Figure 1

Confirmed wild poliovirus

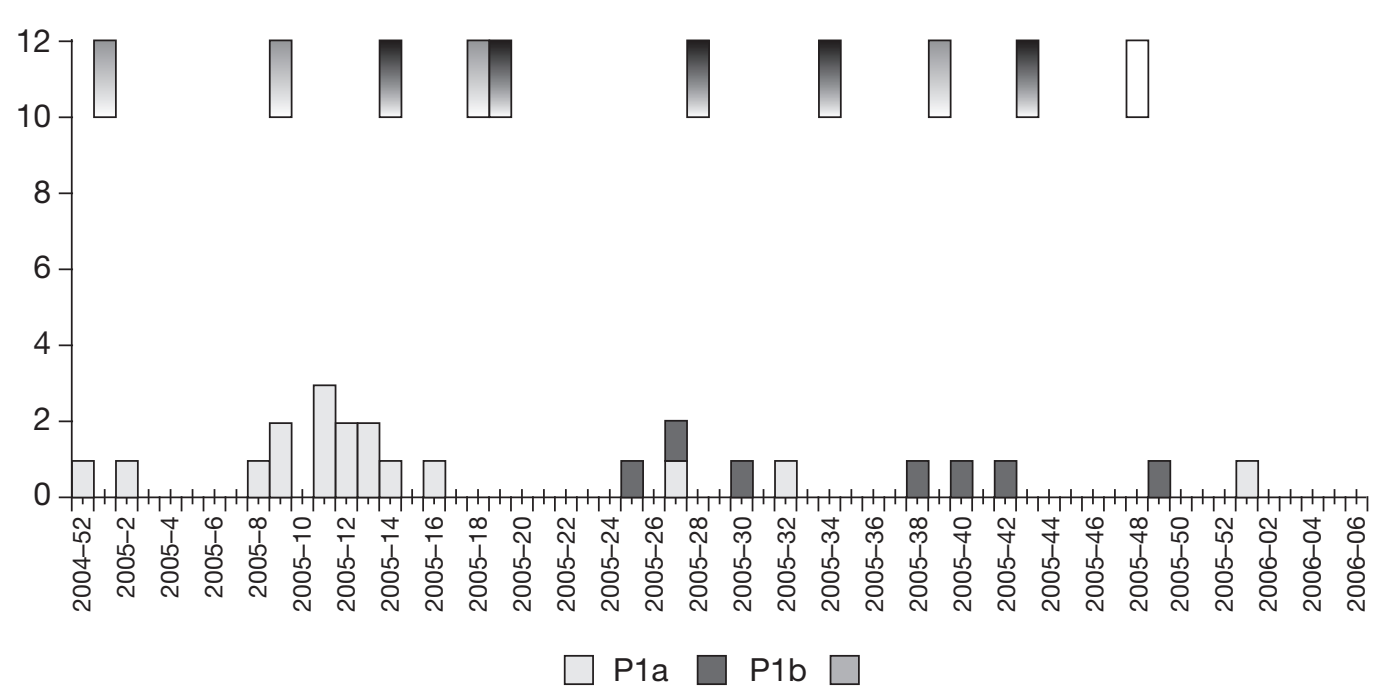

Table 1

Confirmed wild poliovirus cases

\begin{tabular}{|c|c|c|c|c|}
\hline \multirow[b]{2}{*}{ Region } & \multicolumn{4}{|c|}{ Number of confirmed wild polioviruses (all P1) } \\
\hline & District & 2004 & 2005 & 2006 * \\
\hline \multirow[t]{4}{*}{ Tigray } & Tahttay koraro & 1 & & \\
\hline & Kafta Humera & & 5 & \\
\hline & Tsegede & & 1 & \\
\hline & Ofla & & 1 & \\
\hline \multirow[t]{8}{*}{ Amhara } & Tach Armacho & & 1 & \\
\hline & Tegede & & 1 & \\
\hline & Gondar & & 1 & \\
\hline & Fogera & & 1 & \\
\hline & Farta & & 2 & \\
\hline & Bahir Dar & & 1 & \\
\hline & Alefa Taqusa & & 1 & \\
\hline & Sekota & & & 1 \\
\hline \multirow[t]{4}{*}{ Oromia } & Kombelcha & & 2 & \\
\hline & Fedis & & 3 & \\
\hline & Meta & & 1 & \\
\hline & Haro Mayo & & 1 & \\
\hline Total & & 1 & 22 & 1 \\
\hline
\end{tabular}

${ }^{*}$ Latest case as of $1^{\text {st }}$ February 2006 
Table 2

Clinical and epidemiological characteristics of poliomyelitis cases $(n=24)$

\begin{tabular}{|c|c|c|c|c|c|}
\hline \multirow[b]{2}{*}{ By immunisation status } & \multicolumn{4}{|c|}{ Degree of paralysis } & \multirow[b]{2}{*}{ Total } \\
\hline & Monoplegia & Paraplegia & Hemiplegia & Quadriplegia & \\
\hline 0 & 4 & 1 & 0 & 2 & 7 \\
\hline 1 & 4 & 1 & 0 & 1 & 6 \\
\hline 2 & 0 & 0 & 0 & 1 & 1 \\
\hline $3+$ & 6 & 3 & 0 & 1 & 10 \\
\hline Total & 14 & 5 & 0 & 5 & 24 \\
\hline \multicolumn{6}{|c|}{$\begin{array}{l}\text { By age, stratified according } \\
\text { to OPV status }\end{array}$} \\
\hline \multicolumn{6}{|c|}{$0-2$ doses } \\
\hline$<5$ years & 8 & 2 & 0 & 0 & 10 \\
\hline$\geq 5$ years & 0 & 1 & 0 & 3 & 4 \\
\hline \multicolumn{6}{|l|}{$3+$ doses } \\
\hline$<5$ years & 2 & 3 & 0 & 0 & 5 \\
\hline$\geq 5$ years & 1 & 1 & 0 & 3 & 5 \\
\hline Total & 11 & 7 & 0 & 6 & 24 \\
\hline
\end{tabular}

One child who had received four doses of OPV and initially had quadriplegia, on repeat clinical evaluation 60 days after paralysis onset had only minor residual weakness. Fever was associated with the onset of paralysis for all of the paralytic poliomyelitis cases. Of the 24 paralysed children, $14(58 \%)$ had one of their lower limbs paralysed (monoplegia), five $(21 \%)$ had both lower limbs paralysed (paraplegia); and five $(21 \%$ ) had all limbs with flaccid paralysis (quadriplegia).

The age-specific comparison when data was stratified according to immunisation status (Table 2), also shows that of the paralysed children aged below five years who had received less than three doses of OPV, eight out of ten $(80 \%)$ had monoplegia, and only two (20\%) had paraplegia. Whereas, among those aged five years and above, three out of four $(75 \%)$ cases had quadriplegia and only one out of four $(25 \%)$ cases had paraplegia. Moreover, of the 10 paralysed children with at least three doses of OPV, three out of five $(60 \%)$ and one out of five (20\%) of the paralysed children aged above five years had quadriplegia and monoplegia respectively. In those below five years, however, none of the five paralysed children had quadriplegia.

Following confirmation of wild poliovirus infection in the index cases (from each outbreak) and subsequent cases, detailed outbreak investigations were conducted in all three regions within 48 hours of receiving confirmation of the index case. One of the early cases identified in Tigray region had history of recent travel to the neighbouring country of Sudan. Of the 24 cases, $15(63 \%)$ lived in rural villages while nine $(38 \%)$ cases lived in semi-urban settings.

Table 3 summarises the findings of the field investigations that included epidemiological and clinical assessment. During the study period, in total sixty eight AFP cases were reported from the three regions, (27 AFP cases from Tigray, 26 in Amhara and 15 AFP cases in Oromia). The median non-polio AFP rate in the affected districts was 6 (range: 0 to 60 days) per 100,000 children aged less than 15 years. Adequate (Two stool specimens collected at least 24 hours apart and within 14 days of paralysis onset. Each specimen must be of adequate quantity (8-10 g), arrive in good condition (no desiccation, no leakage) and have adequate documentation and evidence that the reverse cold chain was maintained) stool specimens were obtained from $38(73 \%)$ of the 52 AFP cases. Among confirmed WPV cases, however, adequate stool specimens were taken from 22 of the $24(92 \%)$ cases. The median duration from onset to date of investigation of the WPV cases was 8.4 days (range: 2 to 24 days). 
Follow-up visits were carried out on 12 (100\%) of the late reported AFP cases to clinically evaluate for residual paralysis 60 days after onset of acute flaccid paralysis. Eight of the twelve cases with inadequate specimens had residual paralysis at the time of investigation. The eight cases were clinically diagnosed as follows: Guillain-Barre Syndrome (three cases); traumatic neuritis (two cases); transverse myelitis (one case); simple focal seizure with Todd's paralysis (one case); and Pott's disease (one case).

Active case search for unreported AFP cases, which included review of medical records, search during house-to-house visits / interviews, and visits to traditional healing units revealed one unreported AFP case in Tsegede District of Tigray and two in Kombolcha District of Oromia regions. The majority $(86 \%)$ of the district focal persons were not doing independent active case search in their catchment areas. Moreover, health workers in fifty four per cent of the health facilities assessed were not aware of the case definition for acute flaccid paralysis.

The study also disclosed that majority of the child caretakers in the affected areas frequently consulted traditional healing units such as holy water sites, and traditional healers, including bone setters and spiritualists. This contributed to late investigation of AFP cases. Modern health care systems were least cited as the location where children with paralysis would be taken first. Poor sanitation, inadequate water supply and crowded living conditions described the affected communities in all three regions.

The OPV coverage and supplementary immunisation activities were assessed for a sample of 1,017 children aged 12-23 months from eight affected districts in Tigray, Amhara and Oromia regions (Table 3). In 2004 and 2005, the reported administrative coverage of three or more doses of OPV by one year of age was $86 \%$, and $85 \%$ for Tigray, $75 \%$, and $80 \%$ for Amhara and $47 \%$, and $75 \%$ for Oromia regions, respectively. The median routine coverage of at least three doses of OPV (survey) by one year of age in the affected districts, however, was
$74 \%$ (range: $54 \%$ to $98 \%$ ) with coverage in seven of the eleven communities less than $70 \%$. Few $(4.5 \%$; range $2.1 \%$ to $5.2 \%$ ) of the children had received no doses of OPV at 12 months of age. Seventy five per cent of the health facilities, in the affected communities, had either poor vaccine handling or non-functional refrigerators. Eligible children in most of the resettlement sites of Southwest Tigray and North Gondar Zone of Amhara had interrupted immunisation services at the time of the survey.

In response to the outbreak, the country intensified actions to correct gaps in surveillance, and immunisation services in the affected areas. Cascaded training and sensitization was given to all surveillance staff including clinicians. Surveillance focal persons intensified active surveillance in all health facilities including traditional healing sites, such as holy water and traditional healers. Routine vaccination activities were strengthened in the affected communities including resettlement sites. The country implemented five rounds of full-scale national house-to-house and four sub-national polio vaccination campaigns (Figure 4). The median duration between paralysis onset of the index case and the start of the vaccination campaigns was two weeks (range: 1 to 4 weeks). The median coverage of at least one dose of OPV during the National Immunisation Days (NIDs) in February and May 2005 was $100 \%$ (range: 87 to 100). Response measures were regularly monitored in all the affected districts and communities.

Following the scaling up of the response measures, the three regions documented interrupted transmission of wild poliovirus within one year (Figure 4). By the beginning of February 2005 annualised AFP detection rate had improved to above two per one hundred thousand children less than 15 years of age, and adequacy of specimen collection to above $80 \%$ in most except Komboleha (75\%) of Oromia and Fogera (67\%) of Amhara regions. The latest date of onset of paralysis due to the imported wild poliovirus was $1^{\text {st }}$ February 2006. 
Table 3

Results of field investigations in the polio affected districts

\begin{tabular}{|c|c|c|c|c|c|c|c|c|}
\hline \multirow{4}{*}{ Parameter } & \multicolumn{8}{|c|}{ Districts surveyed following confirmation of wild poliovirus infection } \\
\hline & \multicolumn{2}{|c|}{ Tigray } & \multicolumn{2}{|c|}{ Amhara } & & \multicolumn{3}{|c|}{ Oromiya } \\
\hline & Tahtay & Kafta & Gondar & Fogera & Bahir & Kombolcha & Fedis & Meto \\
\hline & koraro & Humera & town & & Dar & & & \\
\hline \multirow[t]{2}{*}{ Population $<15$ years } & 54,233 & 52,320 & 45,500 & 55,231 & 65,200 & 45,231 & 3,623 & 2,432 \\
\hline & & & & & & 2 & 0 & \\
\hline WPVcases & 1 & 5 & 1 & 2 & 1 & 3 & 2 & 2 \\
\hline \multicolumn{9}{|l|}{ AFP cases } \\
\hline Reported & 4 & 18 & 4 & 6 & 8 & 4 & 6 & 5 \\
\hline Identified by active surveillance & 0 & 1 & 0 & 0 & 0 & 2 & 0 & 1 \\
\hline Non-polio AFP rate per 100,000 & 18 & 60 & 4 & 4 & 7 & 7 & 2 & 1 \\
\hline \multicolumn{9}{|l|}{ population aged $<15$ years } \\
\hline Adequacy of stool specimen collection (\%) & 67 & 78 & 100 & 67 & 80 & 75 & 100 & 40 \\
\hline \multicolumn{9}{|l|}{ Two specimens within 2 weeks } \\
\hline \multicolumn{9}{|l|}{ Good condition } \\
\hline \multicolumn{9}{|l|}{ On ice } \\
\hline \multicolumn{9}{|l|}{ Quantity 8-10g } \\
\hline \multicolumn{9}{|l|}{ OPV coverage survey $(\%)$} \\
\hline \multicolumn{9}{|l|}{ Routine immunisation } \\
\hline No dose & 2.1 & 2.5 & 3.5 & 4.5 & NA & 5.6 & 5.2 & 3.4 \\
\hline 1 dose & 82.3 & 96.9 & 98.9 & 100 & 82.6 & 75 & 80.2 & 80.7 \\
\hline 3 doses & 88.8 & 74.0 & 97.8 & 94.7 & 89.3 & 75 & 54.2 & 64.0 \\
\hline \multicolumn{9}{|l|}{ Supplementary immunisation $\geq 1$ dose } \\
\hline in NIDs* / SNIDs & 100 & 98.5 & 100 & 100 & 90 & 94 & 90.3 & 96 \\
\hline
\end{tabular}

${ }^{*}$ NIDs $=$ National Immunisation Days 
Figure 2

Age of confirmed polio cases $(n=24)$

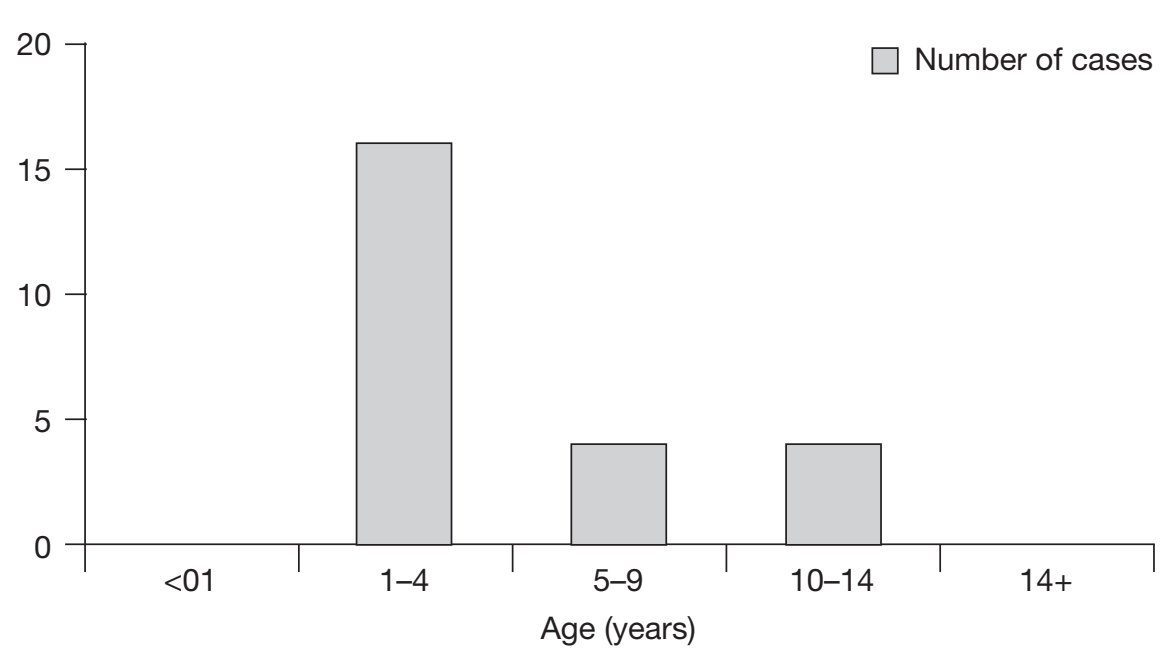

Figure 3

Number of OPV doses among confirmed polio cases $(n=24)$

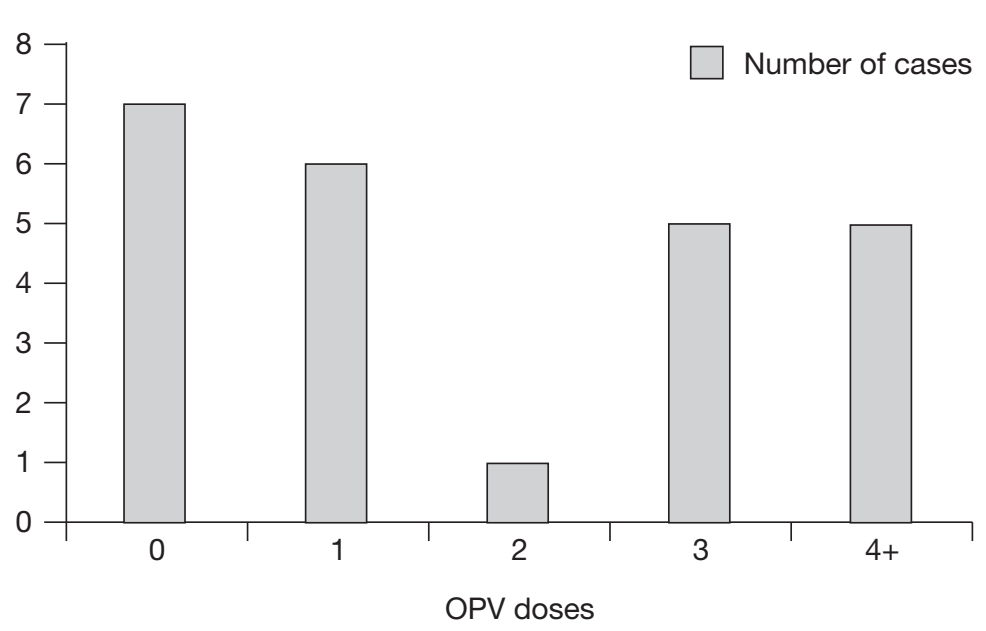

Figure 4

Epidemic curve of poliomyelitis versus supplementary immunisation activities

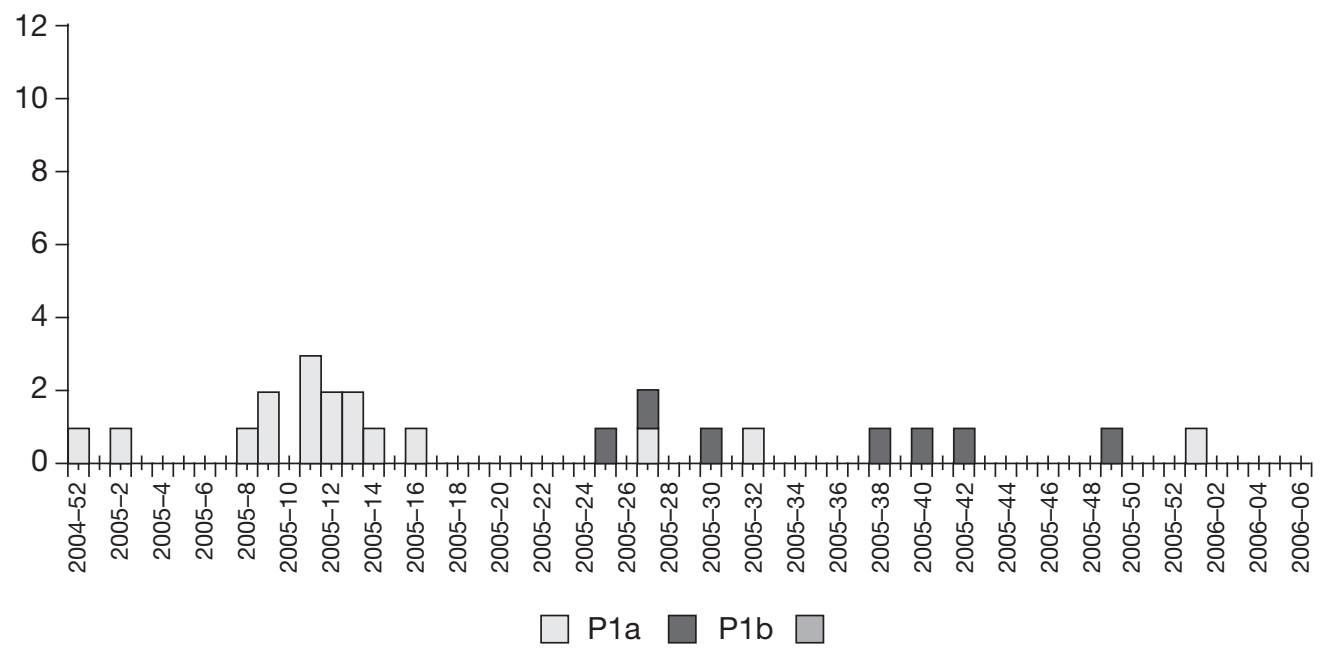




\section{DISCUSSION}

The global effort toward polio eradication has been delayed because of the re-introduction of wild poliovirus to previously polio-free countries. Polio eradication efforts in Ethiopia had been successful, with the last case of indigenous wild poliovirus reported in January $2001(7,8)$. Prompt investigation and response to outbreaks is necessary to contain and prevent re-established circulation of wild poliovirus.

Findings from the present investigation of the polio outbreaks in Ethiopia showed that a total of 24 children, all aged less than 15 years, were paralysed as a result of infection with wild poliovirus. All of the cases suffered paralysis from polio infection. Genetic sequencing results demonstrated that the wild poliovirus isolated from cases in Tigray and Amhara regions was most closely related to the virus isolated in Khartoum, Sudan. Whereas, the wild poliovirus found in East Harerghe of Oromia region, most closely matched wild poliovirus isolated in the Red Sea Province of Sudan. Genetic sequencing from these outbreaks, therefore, demonstrates the presence of at least two separate routes of spread of wild poliovirus to Ethiopia.

Epidemiologic investigation of poliomyelitis, furthermore discloses that $16(66.7 \%)$ of the 24 children with paralysis were less than five years of age. Fourteen out of 24 (58.3\%) paralysed children had received fewer than three doses of OPV. Furthermore, seven $(29.2 \%)$ out of the 24 children with paralysis had never received any dose of OPV. Additionally, the paralysis (paraplegia and quadriplegia) tended to be more severe among nonimmunised and older aged children. This is similar to previous reports from India $(3,4)$.

Combinations of risk factors including programmatic failures presented opportunities for introduction and further spread of the wild virus in the affected districts and communities. Routine OPV3 coverage was less than $80 \%$ in 12 out of 14 affected districts. Additionally, the cold chain system was found to be below the standard in health facilities in the affected districts. In most of the re-settlement areas, in Tigray and Amhara regions, and in East Harerghe, it was noted that eligible children were not reached by routine immunisation services.

Sixty five per cent of the affected communities had inadequate stool specimen collection, as well as inaccurate and incomplete AFP surveillance data. In the affected districts irregular active case search was conducted by focal persons. Community surveillance networks had not been optimally utilised for active AEP case search, particularly the community health workers (community health agents, and traditional birth attendants) and traditional healing units of the affected districts and communities. Crowded living conditions of the settlers in the affected areas, lack of adequate clean water supply, and lack of adequate sanitation were characteristics noted in affected areas.

Ethiopia shares a long border with the Sudan, with Tigray and Amhara regions bordering Sudan where clustering of polio cases had been reported. East Harerghe of Oromia region may have been at increased risk of polio importation due to considerable transborder flow of merchants from Sudan and Djibouti. Farming, trade of goods and services between bordering communities, family ties, availability of health services, and other activities favour the continuous interaction between the populations of these bordering countries.

The outbreak investigations disclosed that multiple risk factors favoured the continuous spread of the wild poliovirus in the affected communities of Tigray, Amhara and East Harerghe of Oromia regions. The combination of weakness in AFP surveillance quality and the low routine immunisation coverage among children in the affected communities favoured the spread of the outbreak. Evaluation of the cold chain system in all health care facilities of the affected districts was found to be below expectation. Additionally, because the last nationwide polio immunisation campaign was conducted in 2002, may have increased the risk for spread of the wild poliovirus.

The effectiveness of the outbreak response activities by the country was satisfactory. Response activities were implemented according to the WHO recommendations (5). The country had attained surveillance standards and interrupted the wild poliovirus transmission in the three regions within one year. This demonstrates the capacity to vaccinate enough of the susceptible children to produce herd immunity.

In conclusion, the 2004-2006 polio outbreaks in the three regions of Tigray, Amhara and Oromia of Ethiopia provides lessons on the possible risks 
for wild poliovirus transmission as a result of importation, from the remaining endemic and reinfected countries. Interruption of the imported wild poliovirus transmission in a short period of time on the other hand demonstrates the significance of enhanced and timely detection and implementation of effective outbreak response activities. Detection of wild poliovirus in a polio-free country is a public health emergency. In order to interrupt the spread of the virus and prevent re-established transmission, it is necessary to correct programmatic failures in the affected areas. Programme strategies need to be reactivated or intensified. To improve the weaknesses in the quality of AFP surveillance and routine vaccination, health workers should be resensitized periodically. No vaccination programme can be successful without an effective routine programme. An endeavour to interrupt transmission and eradicate polio requires strengthening routine immunisation activities with emphasis to reach communities. Additionally, two rounds of high quality nationwide immunisation campaigns have been recommended annually for Horn of Africa countries until polio transmission is interrupted in remaining endemic reservoirs (14).

\section{ACKNOWLEDGEMENTS}

To the district surveillance and routine immunisation experts for their commitment to polio eradication. The authors would also like to acclaim the diligent work of surveillance officers who participated during the polio outbreak investigations.

\section{REFERENCES}

1. Global eradication of poliomyelitis by the year 2000 . Geneva, World Health Organization, 1999 (World Health Assembly Resolution WHA41 .28).
2. Expanded Program on Immunisation. Progress towards global eradication of poliomyelitis, 1997.

3. Expanded Programme on Immunisation. Certification of poliomyelitis eradication-the Americas. Weekly Epidem. Record. 1994; 69: 293-295.

4. Organisation of African Unity. Yaounde declaration on polio eradication in Africa. Proceedings of 32nd Ordinary Session of the Organisation of African Unity meeting. Yaounde, Cameroon: Organisation of African Unity, 1996; AHG/Declaration I (XXXII).

5. Maiga, Z., Kamwa, M., Gasasira, A., et al. Poliomyelitis outbreak in polio-free countries in Central and West Africa. Comm. Dis. Bull. Afr. Region. 2004; 2: 4-6.

6. WHO. Guidelines on response to suspected outbreaks of poliomyelitis, Harare, June 2004.

7. Centre for Diseases Control. Progress towards poliomyelitis eradication -Ethiopia, 1997-August 2000. MMWR. 2000; 49: 867-870.

8. Ethiopia Federal Ministry of Health. The national routine immunisation update, June 2005.

9. World Health Organisation/United Nations Children Fund. Review of national immunisation coverage 1980-2003. 2004.

10. Kaushik, B., Hlday, W.G., Andrus, J.K., et al. Poliomyelitis surveillance: the model used in India for polio eradication. Bull. WHO. 2000; 78: 321-329.

11. Hoekstra, E.J., Feng, C., Xiao-jun, Bilous, J., et al. Excluding polio in areas of inadequate surveillance in the final stages of eradication in china. Bull. WHO. 2000; 78: 315-320.

12. Morgan, J.W. Principles of epidemiology: A practical text. First edition. Loma Linda, 2000.

13. Dean, A., Arner, T.G., Sangam, S., et al. Epi Info, version 3.2.2. A database and statistics program for public health professionals for use on Windows 95, 98, NT and 2000 computers. Centers for Disease Control and Prevention, Atlanta, Georgia, USA, 2002.

14. Horn of Africa Technical Advisory Group Recommendations, August 2006. 